\title{
Partial Extinction, Permanence, and Global Attractivity in Nonautonomous $n$-Species Gilpin-Ayala Competitive Systems with Impulses
}

\author{
Juan Hou and Hanhui Liu \\ College of Applied Mathematics, Xinjiang University of Finance and Economics, Urumqi 830012, China \\ Correspondence should be addressed to Juan Hou, hj_xd@yahoo.com.cn \\ Received 3 May 2012; Accepted 21 June 2012 \\ Academic Editor: Yong Zhou \\ Copyright (c) 2012 J. Hou and H. Liu. This is an open access article distributed under the Creative \\ Commons Attribution License, which permits unrestricted use, distribution, and reproduction in \\ any medium, provided the original work is properly cited. \\ The qualitative properties of general nonautonomous n-species Gilpin-Ayala competitive systems \\ with impulsive effects are studied. Some new criteria on the permanence, extinction, and global \\ attractivity of partial species are established by using the methods of inequalities estimate and \\ Liapunov functions.
}

\section{Introduction}

In [1], the general nonautonomous $n$-species Lotka-Volterra competitive systems with impulsive effects are investigated. By using the methods of inequalities estimate and constructing the suitable Liapunov functions, the sufficient conditions on the permanence of whole species and global attractivity of systems are established.

In [2], the authors studied the following general nonautonomous $n$-species LotkaVolterra competitive systems with impulsive perturbations:

$$
\begin{gathered}
\dot{x}_{i}(t)=x_{i}(t)\left[a_{i}(t)-\sum_{j=1}^{n} b_{i j}(t) x_{j}(t)\right], \quad t \neq t_{k}, \\
x_{i}\left(t_{k}^{+}\right)=h_{i k} x_{i}\left(t_{k}\right), \quad k=1,2, \ldots, i=1,2, \ldots, n,
\end{gathered}
$$

and got a series of criteria on the extinction of a part of $n$-species, the permanence of other part of $n$-species, and the global attractivity of the systems. 
In [3], a periodic $n$-species Gilpin-Ayala competition system with impulses is studied and obtain some useful behaviors of the system.

In this paper, we investigate the general nonautonomous n-species Gilpin-Ayala competitive systems with impulsive effects.

$$
\begin{aligned}
& \dot{x}_{i}(t)=x_{i}(t)\left[a_{i}(t)-\sum_{j=1}^{n} b_{i j}(t) x_{j}^{\theta_{i j}}(t)\right], \quad t \neq t_{k}, \\
& x_{i}\left(t_{k}^{+}\right)=h_{i k} x_{i}\left(t_{k}\right), \quad k=1,2, \ldots, i=1,2, \ldots, n,
\end{aligned}
$$

where $b_{i}(t)$ and $a_{i j}(t)(i, j=1,2, \ldots, n)$ are defined on $R_{+}=[0, \infty)$ and are bounded continuous functions, $a_{i j}(t) \geq 0$ for all $t \in R_{+}, \theta_{i j}$ and $h_{i k}>0$ are constants for all $k=1,2, \ldots$ and $i, j=1,2, \ldots, n$.

\section{Preliminaries}

Firstly, we introduce the following assumption.

Assumption $H$. There is a positive constant $\omega$ such that for each $i=1,2, \ldots, n$

$$
\liminf _{t \rightarrow \infty} \int_{t}^{t+\omega} a_{i i}(s) d s>0, \quad \liminf _{t \rightarrow \infty}\left(\int_{t}^{t+\omega} b_{i}(s) d s+\sum_{t \leqslant t_{k} \leqslant t+\mu} \ln h_{i k}\right)>0,
$$

and functions

$$
h_{i}(t, \mu)=\sum_{t \leqslant t_{k} \leqslant t+\mu} \ln h_{i k}, \quad i=1,2, \ldots, n
$$

are bounded on $t \in R_{+}$and $0 \leqslant \mu \leqslant \omega$.

For each $i \in\{1,2, \ldots, n\}$, we consider the following logistic impulsive equation as the subsystem of system (1.2)

$$
\begin{gathered}
\dot{x}_{i}(t)=x_{i}(t)\left[b_{i}(t)-a_{i i}(t) x_{i}^{\theta_{i i}}(t)\right], \quad t \neq t_{k} \\
x_{i}\left(t_{k}^{+}\right)=h_{i k} x_{i}\left(t_{k}\right), \quad k=1,2, \ldots
\end{gathered}
$$

From the above assumption, we have the following results. 
Lemma 2.1. Suppose that assumption $H$ holds. Then we have the following:

(1) There exist positive constants $m$ and $M$ such that

$$
m \leqslant \liminf _{t \rightarrow \infty} x_{i}(t) \leqslant \limsup _{t \rightarrow \infty} x_{i}(t) \leqslant M,
$$

for any positive solution $u_{i}(t)$ of (2.3).

(2) $\lim _{t \rightarrow \infty}\left(x_{i}^{(1)}(t)-x_{i}^{(2)}(t)\right)=0$ for any two positive solutions $x_{i}^{(1)}(t)$ and $x_{i}^{(2)}(t)$ of (2.3).

Proof. From assumption $\mathrm{H}$, there are positive constants $k_{1}, k_{2}, \delta$ and $T_{0}$ such that for all $t \geqslant T_{0}$ we have

$$
\begin{aligned}
& \int_{t}^{t+\omega}\left(b_{i}(s)-a_{i i}(s) k_{1}\right) d s+\sum_{t \leq t_{k}<t+\omega} \ln h_{k}<-\delta, \\
& \int_{t}^{t+\omega}\left(b_{i}(s)-a_{i i}(s) k_{2}\right) d s+\sum_{t \leq t_{k}<t+\omega} \ln h_{k}>\delta .
\end{aligned}
$$

From the boundedness of function $h(t, \mu)=\sum_{t \leq t_{k}<t+\mu} \ln h_{k}$, there is a positive constant $P$ such that for any $t \in R_{+}$and $\mu \in[0, \omega)$

$$
|h(t, \mu)|=\left|\sum_{t \leq t_{k}<t+\mu} \ln h_{k}\right|<P .
$$

Firstly, we prove that there is a constant $M>0$ such that

$$
\limsup _{t \rightarrow \infty} x_{i}(t)<M,
$$

for any positive solution $x_{i}(t)$ of system (2.3). In fact, for any positive solution $x_{i}(t)$ of system (2.3), we only need to consider the following three cases.

Case I. There is a $t_{0} \geq T_{0}$ such that $x(t) \geq k_{1}^{\prime}=\sqrt[\theta_{i i}]{k_{1}}$ for all $t \geq t_{0}$.

Case II. There is a $t_{0} \geq T_{0}$ such that $x(t) \leq k_{1}^{\prime}$ for all $t \geq t_{0}$.

Case III. $x(t)$ is oscillatory about $k_{1}^{\prime}$ for all $t \geq T_{0}$. 
We first consider Case I. Since $x_{i}(t) \geq k_{1}^{\prime}$ for all $t \geq t_{0}$, then for $t=t_{0}+l \omega$, where $l \geq 0$ is any positive integer, integrating system (2.3) from $t_{0}$ to $t$, from (2.5) we have

$$
\begin{aligned}
x_{i}(t)=x_{i}\left(t_{0}\right) \exp \left(\int_{t_{0}}^{t}\left(b_{i}(s)-a_{i i}(s) x_{i}^{\theta_{i i}}(s)\right) d s+\sum_{t_{0} \leq t_{k}<t} \ln h_{k}\right) \\
\leq x_{i}\left(t_{0}\right) \exp \left(\int_{t_{0}}^{t_{0}+\omega}\left(b_{i}(s)-a_{i i}(s) k_{1}\right) d s+\sum_{t_{0} \leq t_{k}<t} \ln h_{k}+\cdots\right. \\
\left.+\int_{t_{0}+(l-1) \omega}^{t_{0}+l \omega}\left(b_{i}(s)-a_{i i}(s) k_{1}\right) d s+\sum_{t_{0}+(l-1) \omega \leq t_{k}<t_{0}+l \omega} \ln h_{k}\right) \\
\leq x_{i}\left(t_{0}\right) \exp (-l \delta) .
\end{aligned}
$$

Hence, $x_{i}(t) \rightarrow 0$ as $l \rightarrow \infty$, which leads a contradiction.

Next, we consider Case III. From the oscillation of $x_{i}(t)$ about $k_{1}^{\prime}$, we can choose two sequences $\left\{\rho_{n}\right\}$ and $\left\{\rho_{n}^{*}\right\}$ satisfying $T_{0}<\rho_{1}<\rho_{1}^{*}<\cdots<\rho_{n}<\rho_{n}^{*}<\cdots$ and $\lim _{n \rightarrow \infty} \rho_{n}=$ $\lim _{n \rightarrow \infty} \rho_{n}^{*}=\infty$ such that

$$
\begin{gathered}
x_{i}\left(\rho_{n}\right) \leq k_{1}^{\prime}, \quad x_{i}\left(\rho_{n}^{+}\right) \geq k_{1}^{\prime}, \quad x_{i}\left(\rho_{n}^{*}\right) \geq k_{1}^{\prime}, \quad x_{i}\left(\rho_{n}^{*^{+}}\right) \leq k_{1}^{\prime}, \\
x_{i}(t) \geq k_{1}^{\prime}, \quad \forall t \in\left(\rho_{n}, \rho_{n}^{*}\right), \\
x_{i}(t) \leq k_{1}^{\prime}, \quad \forall t \in\left(\rho_{n}^{*}, \rho_{n+1}\right) .
\end{gathered}
$$

For any $t \geq T_{0}$, if $t \in\left(\rho_{n}, \rho_{n}^{*}\right]$ for some integer $n$, then we can choose integer $l \geq 0$ and constant $0 \leq \mu_{1}<\omega$ such that $t=\rho_{n}+l \omega+\mu_{1}$. Since

$$
\dot{x}_{i}(t) \leq x_{i}(t)\left(b_{i}(t)-a_{i i}(t) k_{1}\right), \quad \forall t \in\left(\rho_{n}, \rho_{n}^{*}\right), \quad t \neq t_{k},
$$


integrating this inequality from $\rho_{n}$ to $t$, by (2.5) and (2.7) we obtain

$$
\begin{aligned}
x_{i}(t)= & x_{i}\left(\rho_{n}\right) \exp \left(\int_{\rho_{n}}^{t}\left(b_{i}(s)-a_{i i}(s) x^{\theta_{i i}}(s)\right) d s+\sum_{\rho_{n} \leq t_{k}<t} \ln h_{k}\right) \\
\leq & k_{1}^{\prime} \exp \left(\int_{\rho_{n}}^{\rho_{n}+\omega}\left(b_{i}(s)-a_{i i}(s) k_{1}\right) d s+\sum_{\rho_{n} \leq t_{k}<\rho_{n}+\omega} \ln h_{k}+\cdots\right. \\
& \left.+\int_{\rho_{n}+l \omega}^{\rho_{n}+l \omega+\mu_{1}}\left(b_{i}(s)-a_{i i}(s) k_{1}\right) d s+\sum_{\rho_{n}+l \omega \leq t_{k}<\rho_{n}+l \omega+\mu_{1}} \ln h_{k}\right) \\
\leq & k_{1}^{\prime} \exp \left(-l \delta+\int_{\rho_{n}+l \omega}^{\rho_{n}+l \omega+\mu_{1}}\left(b_{i}(s)-a_{i i}(s) k_{1}\right) d s+\sum_{\rho_{n}+l \omega \leq t_{k}<\rho_{n}+l \omega+\mu_{1}} \ln h_{k}\right) \\
\leq & k_{1}^{\prime} \exp \left(\alpha_{1} \omega+P\right),
\end{aligned}
$$

where $\alpha_{1}=\sup _{t \in R_{+}}\left\{\left|b_{i}(t)\right|+a_{i i}(t) k_{1}\right\}$. If there is an integer $n$ such that $t \in\left(\rho_{n}^{*}, \rho_{n+1}\right]$, then we obviously have

$$
x_{i}(t) \leq k_{1}^{\prime}<k_{1}^{\prime} \exp \left(\alpha_{1} \omega+P\right)
$$

Therefore, for Case III we always have

$$
x_{i}(t) \leq k_{1}^{\prime} \exp \left(\alpha_{1} \omega+P\right), \quad \forall t \geq T_{0}
$$

Lastly, if Case II holds, then we directly have

$$
x_{i}(t) \leq k_{1}^{\prime} \exp \left(\alpha_{1} \omega+P\right), \quad \forall t \geq T_{0}
$$

Choose constant $M=k_{1}^{\prime} \exp \left(\alpha_{1} \omega+P\right)$, then we see that (2.8) holds.

Secondly, a similar argument as in the proof of (2.8) we can prove that there is a constant $m>0$, such that

$$
\liminf _{t \rightarrow \infty} x(t)>m,
$$

for any positive solution $x_{i}(t)$ of system (2.3). Conclusion (1.1) is proved.

Now, we prove conclusion (1.2). Let $x_{i}^{(1)}(t)$ and $x_{i}^{(2)}(t)$ be any two positive solutions of system (2.3). From conclusion (1.1), it follows that there are positive constants $A$ and $B$ such that

$$
A \leqslant x_{i}^{(1)}(t), \quad x_{i}^{(2)}(t) \leqslant B, \quad \forall t \geq 0 .
$$


Choose Liapunov function as follows:

$$
V(t)=\left|\ln x_{i}^{(1)}(t)-\ln x_{i}^{(2)}(t)\right|
$$

For any $k=1,2, \ldots$, we have

$$
V\left(t_{k}^{+}\right)=\left|\ln \left(h_{k} x_{i}^{(1)}\left(t_{k}\right)\right)-\ln \left(h_{k} x_{i}^{(2)}\left(t_{k}\right)\right)\right|=V\left(t_{k}\right)
$$

Hence, $V(t)$ is continuous for all $t \in R_{+}$and from the Mean-Value Theorem we can obtain

$$
\frac{1}{B}\left|x_{i}^{(1)}(t)-x_{i}^{(2)}(t)\right| \leqslant V(t) \leqslant \frac{1}{A}\left|x_{i}^{(1)}(t)-x_{i}^{(2)}(t)\right|
$$

Calculating the upper right derivative of $V(t)$, then from (2.20) we obtain

$$
\begin{aligned}
D^{+} V & =\operatorname{sign}\left(x_{i}^{(1)}(t)-x_{i}^{(2)}(t)\right)\left(\frac{\dot{x}_{i}^{(1)}(t)}{x_{i}^{(1)}(t)}-\frac{\dot{x}_{i}^{(2)}(t)}{x_{i}^{(2)}(t)}\right) \\
& =-a_{i i}(t)\left|x_{i}^{(1) \theta_{i i}}(t)-x_{i}^{(2) \theta_{i i}}(t)\right| \\
& \leq-a_{i i}(t)\left[\theta_{i i}\right] A_{i i}^{\theta}\left|x_{i}^{(1)}(t)-x_{i}^{(2)}(t)\right| \\
& \leq-a_{i i}(t)\left[\theta_{i i}\right] A^{\theta_{i i}} V(t), \quad t \neq t_{k}, k=1,2, \ldots,
\end{aligned}
$$

where $\left[\theta_{i i}\right] \leq \theta_{i i}$ is the integer part of $\theta_{i i}$.

From this, we further have for any $t>0$

$$
V(t) \leqslant V(0) \exp \left(-\left[\theta_{i i}\right] A^{\theta_{i i}} \int_{0}^{t} a_{i i}(s) d s\right) .
$$

From condition (2.5) we can obtain $\int_{0}^{t} a_{i i}(t) d t \rightarrow \infty$ as $t \rightarrow \infty$. Hence, $V(t) \rightarrow 0$ as $t \rightarrow \infty$. Further from (2.20) we finally obtain $\lim _{t \rightarrow \infty}\left(x_{i}^{(1)}(t)-x_{i}^{(2)}(t)\right)=0$. Conclusion (1.2) is proved. This completes the proof of Lemma 2.1.

Applying Lemma 2.1 and the comparison theorem of impulsive differential equations, we easily prove the following result.

Lemma 2.2. Suppose that assumption $H$ holds then there is a constant $B>0$ such that

$$
\limsup _{t \rightarrow \infty} x_{i}(t) \leq B, \quad i=1,2, \ldots, n
$$

for any positive solution $x(t)=\left(x_{1}(t), x_{2}(t), \ldots, x_{n}(t)\right)$ of system (1.2). 


\section{Extinction}

On the partial extinction of system (1.2), we have the following result.

Theorem 3.1. Suppose that assumption $H$ holds. Let $r$ be a given integer and $1 \leq r<n$. If for any $l>r$ there is a $i_{l}<l$ such that for any $j \leq l$

$$
\begin{gathered}
\theta_{i_{l} j}=\theta_{l j} \\
\limsup _{t \rightarrow \infty} \frac{\int_{t}^{t+\omega} b_{l}(s) d s+\sum_{t \leqslant t_{k}<t+\omega} \ln h_{l k}}{\int_{t}^{t+\omega} b_{i_{l}}(s) d s+\sum_{t \leqslant t_{k}<t+\omega} \ln h_{i_{l} k}}<\liminf _{t \rightarrow \infty} \frac{a_{l j}(t)}{a_{i_{i j}}(t)}, \quad \forall j \leq l,
\end{gathered}
$$

or

$$
\liminf _{t \rightarrow \infty} \frac{\int_{t}^{t+\omega} b_{i_{l}}(s) d s+\sum_{t \leqslant t_{k}<t+\omega} \ln h_{i_{l} k}}{\int_{t}^{t+\omega} b_{l}(s) d s+\sum_{t \leqslant t_{k}<t+\omega} \ln h_{l k}}>\limsup _{t \rightarrow \infty} \frac{a_{i_{j} j}(t)}{a_{l j}(t)}, \quad \forall j \leq l,
$$

then species $x_{i}(i=r+1, r+2, \ldots, n)$ are extinction, that is, for any positive solution $x(t)=$ $\left(x_{1}(t), x_{2}(t), \ldots, x_{n}(t)\right)$ of system $(1.2)$,

$$
\lim _{t \rightarrow \infty} x_{i}(t)=0, \quad i=r+1, r+2, \ldots, n
$$

Proof. Firstly, from assumption $\mathrm{H}$, that (2.7) still holds and there are constants $\eta_{0}>0$ and $T_{0}>0$ such that

$$
\int_{t}^{t+\omega} b_{i}(s) d s+\sum_{t \leqslant t_{k}<t+\omega} \ln h_{i k} \geq \eta_{0}
$$

for all $t \geq T_{0}$ and $i=1,2, \ldots, n$.

We first prove $x_{n}(t) \rightarrow 0$ as $t \rightarrow \infty$. Without loss of generality, we assume that condition (3.2) holds. When condition (3.3) holds, a similar argument can be given. Since

$$
\limsup _{t \rightarrow \infty} \frac{\int_{t}^{t+\omega} b_{n}(s) d s+\sum_{t \leqslant t_{k}<t+\omega} \ln h_{n k}}{\int_{t}^{t+\omega} b_{p}(s) d s+\sum_{t \leqslant t_{k}<t+\omega} \ln h_{p k}}<\liminf _{t \rightarrow \infty} \frac{a_{n j}(t)}{a_{p j}(t)}, \quad j=1,2, \ldots, n
$$

where $p=i_{n}$. Hence, we can choose positive constants $\alpha, \beta, \varepsilon$ and $T_{n} \geq T_{0}$ such that

$$
\frac{\int_{t}^{t+\omega} b_{n}(s) d s+\sum_{t \leqslant t_{k}<t+\omega} \ln h_{n k}}{\int_{t}^{t+\omega} b_{p}(s) d s+\sum_{t \leqslant t_{k}<t+\omega} \ln h_{p k}}<\frac{\alpha}{\beta}-\varepsilon<\frac{\alpha}{\beta}<\frac{a_{n j}(t)}{a_{p j}(t)}
$$


for all $t \geq T_{n}$ and $j=1,2, \ldots, n$. Hence, from (3.5) we further obtain

$$
\begin{aligned}
& \int_{t}^{t+\omega}\left(-\alpha b_{p}(s)+\beta b_{n}(s)\right) d s+\beta \sum_{t \leq t_{k}<t+\omega} \ln h_{n k}-\alpha \sum_{t \leq t_{k}<t+\omega} \ln h_{p k} \\
& <-\beta \varepsilon\left(\int_{t}^{t+\omega} b_{p}(s) d s+\sum_{t \leq t_{k}<t+\omega} \ln h_{p k}\right) \\
& \leq-\beta \varepsilon \eta_{0}, \\
& \quad \alpha a_{p j}(t)-\beta a_{n j}(t)=\beta a_{p j}\left[\frac{\alpha}{\beta}-\frac{a_{n j}(t)}{a_{p j}(t)}\right]<0,
\end{aligned}
$$

for all $t \geq T_{n}$ and $j=1,2, \ldots, n$.

Consider the Liapunov function as follows:

$$
V_{n}(t)=\left(x_{p}(t)\right)^{-\alpha}\left(x_{n}(t)\right)^{\beta}
$$

Calculating the derivative, and from (3.1), we can obtain for any $t \geq 0$

$$
\begin{aligned}
\frac{d V_{n}(t)}{d t} & =V_{n}(t)\left[-\alpha\left(b_{p}(t)-\sum_{j=1}^{n} a_{p j}(t) x_{j}^{\theta_{p j}}(t)\right)+\beta\left(b_{n}(t)-\sum_{j=1}^{n} a_{n j}(t) x_{j}^{\theta_{n j}}(t)\right)\right] \\
& =V_{n}(t)\left[-\alpha b_{p}(t)+\beta b_{n}(t)+\sum_{j=1}^{n}\left(\alpha a_{p j}(t)-\beta a_{n j}(t)\right) x_{j}^{\theta_{p j}}(t)\right]
\end{aligned}
$$

for all $t \neq t_{k}$ and

$$
V_{n}\left(t_{k}^{+}\right)=h_{p k}^{-\alpha} h_{n k}^{\beta} V_{n}\left(t_{k}\right)
$$

for all $k=1,2, \ldots$. From (3.9), we further have

$$
\begin{gathered}
\frac{d V_{n}(t)}{d t} \leq V_{n}(t)\left(-\alpha b_{p}(t)+\beta b_{n}(t)\right), \quad t \geq T_{n}, \quad t \neq t_{k}, \\
V_{n}\left(t_{k}^{+}\right)=h_{p k}^{-\alpha} h_{n k}^{\beta} V_{n}\left(t_{k}\right), \quad k=1,2, \ldots
\end{gathered}
$$


For any $t>T_{n}$, there is an integer $q_{t} \geq 0$ such that $t \in\left[T_{n}+q_{t} \omega, T_{n}+\left(q_{t}+1\right) \omega\right]$. Hence, by integrating (3.13) from $T_{n}$ to $t$, we obtain

$$
\begin{aligned}
V_{n}(t) \leq V_{n}\left(T_{n}\right) \exp \left(\int_{T_{n}}^{t}\left[-\alpha b_{p}(s)+\beta b_{n}(s)\right] d s+\sum_{T_{n} \leq t_{k}<t} \ln \left(h_{p k}^{-\alpha} h_{n k}^{\beta}\right)\right) \\
=V_{n}\left(T_{n}\right) \exp \left\{\int_{T_{n}}^{T_{n}+\omega}\left[-\alpha b_{p}(s)+\beta b_{n}(s)\right] d s\right. \\
\quad+\sum_{T_{n} \leq t_{k}<T_{n}+\omega} \ln \left(h_{p k}^{-\alpha} h_{n k}^{\beta}\right)+\cdots+\int_{T_{n}+q_{t} \omega}^{t}\left[-\alpha b_{p}(s)+\beta b_{n}(s)\right] d s \\
\left.\quad+\sum_{T_{n}+q_{t} \omega \leq t_{k}<t} \ln \left(h_{p k}^{-\alpha} h_{n k}^{\beta}\right)\right\} \\
\leq M_{n} \exp \left(-\varepsilon \beta \eta_{0} q_{t}\right),
\end{aligned}
$$

where

$$
M_{n}=V_{n}\left(T_{n}\right) \exp \left(\omega \sup _{t \geq 0}\left\{\alpha\left|b_{p}(t)\right|+\beta\left|b_{n}(t)\right|\right\}+(\alpha+\beta) P\right) .
$$

Since $q_{t} \rightarrow \infty$ as $t \rightarrow \infty$, it follows that from (3.14)

$$
V_{n}(t) \longrightarrow 0 \quad \text { as } t \longrightarrow \infty
$$

Since

$$
\begin{gathered}
\left(x_{n}(t)\right)^{\beta}=V_{n}(t)\left(x_{p}(t)\right)^{\alpha}, \\
\left(h_{n k} x_{n}\left(t_{k}\right)\right)^{\beta}=h_{p k}^{\alpha} x_{p}\left(t_{k}\right) h_{p k}^{-\alpha} h_{n k}^{\beta} V_{n}\left(t_{k}\right),
\end{gathered}
$$

by the boundedness of $x(t)$ on $[0, \infty)$ (see Lemma 2.2), we have

$$
x_{n}(t) \longrightarrow 0 \text { as } t \longrightarrow \infty
$$


For any integer $l>r$, assume that we have obtained $x_{i}(t) \rightarrow 0$ as $t \rightarrow \infty$ for all $i>l$. Now, we prove that $x_{l}(t) \rightarrow 0$ as $t \rightarrow \infty$. Suppose that condition (3.3) holds. When condition (3.2) holds, the argument is similar. Let $i=i_{l}$, by (3.1), we have $\theta_{i l j}=\theta_{l j}$, then for $j \leq l$, we have $\theta_{i j}=\theta_{l j}$. Then we can choose positive constants $\lambda, \eta, \delta$ and $T_{l} \geq T_{0}$ such that

$$
\frac{\int_{t}^{t+\omega} b_{q}(s) d s+\sum_{t \leqslant t_{k}<t+\omega} \ln h_{q k}}{\int_{t}^{t+\omega} b_{l}(s) d s+\sum_{t \leqslant t_{k}<t+\omega} \ln h_{l k}}>\frac{\lambda}{\eta}+\delta>\frac{\lambda}{\eta}>\frac{a_{q j}(t)}{a_{l j}(t)},
$$

for all $t \geq T_{l}$, and $j=1,2, \ldots, l$, where $q=i_{l}$.

Consider the Liapunov function as follows:

$$
V_{l}(t)=\left(x_{q}(t)\right)^{-\eta}\left(x_{l}(t)\right)^{\lambda}
$$

By calculating, we obtain for any $t \geq 0$

$$
\begin{array}{r}
\frac{d V_{l}(t)}{d t}=V_{l}(t)[- \\
-\eta b_{q}(t)+\lambda b_{l}(t)+\sum_{j=1}^{l}\left(\eta a_{q j}(t)-\lambda a_{l j}(t)\right) x_{j}^{\theta_{l j}}(t) \\
\left.+\sum_{j=l+1}^{n} \eta a_{q j}(t) x_{j}^{\theta_{q j}}(t)-\sum_{j=l+1}^{n} \lambda a_{l j}(t) x_{j}^{\theta_{l j}}(t)\right]
\end{array}
$$

for all $t \neq t_{k}$ and

$$
V_{l}\left(t_{k}^{+}\right)=h_{q k}^{-\eta} h_{l k}^{\curlywedge} V_{l}\left(t_{k}\right),
$$

for all $k=1,2, \ldots$ From (3.3) and (3.19), we have

$$
\begin{gathered}
\int_{t}^{t+\omega}\left(-\eta b_{q}(s)+\lambda b_{l}(s)\right) d s+\lambda \sum_{t \leq t_{k}<t+\omega} \ln h_{l k}-\eta \sum_{t \leq t_{k}<t+\omega} \ln h_{q k} \\
<-\eta \delta\left(\int_{t}^{t+\omega} b_{l}(s) d s+\sum_{t \leq t_{k}<t+\omega} \ln h_{l k}\right) \\
\leq-\delta \eta \eta_{0}, \quad \\
\eta a_{q j}(t)-\lambda a_{l j}(t)<0,
\end{gathered}
$$


for all $t \geq T_{l}$ and $j=1,2, \ldots, l$. Hence, from (3.21), it follows that

$$
\begin{gathered}
\frac{d V_{l}(t)}{d t} \leq V_{l}(t)\left[-\eta b_{q}(t)+\lambda b_{l}(t)+\sum_{j=1}^{l}\left(\eta a_{q j}(t)-\lambda a_{l j}(t)\right) x_{j}^{\theta_{l j}}(t)\right. \\
\left.+\sum_{j=l+1}^{n} \eta a_{q j}(t) x_{j}^{\theta_{q j}}(t)-\sum_{j=l+1}^{n} \lambda a_{l j}(t) x_{j}^{\theta_{l j}}(t)\right], \quad t \geq T_{l}, \quad t \neq t_{k}, \\
V_{n}\left(t_{k}^{+}\right)=h_{p k}^{-\alpha} h_{n k}^{\beta} V_{n}\left(t_{k}\right), \quad k=1,2, \ldots
\end{gathered}
$$

Since $x_{i}(t) \rightarrow 0$ as $t \rightarrow \infty$ for all $i>l$, by the boundedness of $a_{i j}(t)(i, j=1,2, \ldots, n)$ on $[0, \infty)$, we obtain

$$
\lim _{t \rightarrow \infty} \int_{t}^{t+\omega} \sum_{j=l+1}^{n}\left(\eta a_{q j}(s) x_{j}^{\theta_{q j}}(s)-\lambda a_{l j}(s)\right) x_{j}^{\theta_{l j}}(s) d s=0 .
$$

Hence, for any small $\varepsilon>0$, there is a $T_{l}^{\prime}>0$, such that

$$
\int_{t}^{t+\omega} \sum_{j=l+1}^{n}\left(\eta a_{q j}(s) x_{j}^{\theta_{q j}}(s)-\lambda a_{l j}(s)\right) x_{j}^{\theta_{l j}}(s) d s<\varepsilon, \quad t>T_{l}^{\prime} .
$$

Combining (3.23), it follows that there is enough large $T_{l}^{*}>\max \left\{T_{l}, T_{l}^{\prime}\right\}$ such that for all $t \geq T_{l}^{*}$,

$$
\begin{gathered}
\int_{t}^{t+\omega}\left[-\eta b_{q}(s)+\lambda b_{l}(s)+\sum_{j=l+1}^{n}\left(\eta a_{q j}(s) x_{j}^{\theta_{q j}}(s)-\sum_{j=l+1}^{n} \lambda a_{l j}(s)\right) x_{j}^{\theta_{l j}}(s)\right] d s \\
-\eta \sum_{t \leq t_{k}<t+\omega} \ln h_{q k}+\lambda \sum_{t \leq t_{k}<t+\omega} \ln h_{l k} \leq-\frac{1}{2} \delta \eta \eta_{0}, \\
x_{i}(t) \leq \delta \quad \forall i>l .
\end{gathered}
$$


For any $t>T_{l}^{*}$, we firstly choose an integer $q_{t} \geq 0$ such that $t \in\left(T_{l}^{*}+q_{t} \omega, T_{l}^{*}+\left(q_{t}+1\right) \omega\right]$. Integrating (3.25) from $T_{l}^{*}$ to $t$, then from (3.3) and (3.28), we have

$$
\begin{aligned}
& V_{l}(t) \leq V_{l}\left(T_{l}^{*}\right) \exp \left\{\int_{T_{l}^{*}}^{t}\left[-\eta b_{q}(s)+\lambda b_{l}(s)+\sum_{j=l+1}^{n}\left(\eta a_{q j}(s) x_{j}^{\theta_{q j}}(s)-\sum_{j=l+1}^{n} \lambda a_{l j}(s)\right) x_{j}^{\theta_{l j}}(s)\right] d s\right. \\
& \left.+\sum_{T_{l}^{*} \leq t_{k}<t} \ln \left(h_{q k}^{-\eta} h_{l k}^{\lambda}\right)\right\} \\
& =V_{l}\left(T_{l}^{*}\right) \exp \left\{\left(\int_{T_{l}^{*}}^{T_{l}^{*}+\omega}\left[-\eta b_{q}(s)+\lambda b_{l}(s)+\sum_{j=l+1}^{n} \eta a_{q j}(s) x_{j}^{\theta_{q j}}(s)-\sum_{j=l+1}^{n} \lambda a_{l j}(s) x_{j}^{\theta_{l j}}(s)\right] d s\right.\right. \\
& \left.+\sum_{T_{l}^{*} \leq t_{k}<T_{l}^{*}+\omega} \ln \left(h_{q k}^{-\eta} h_{l k}^{\lambda}\right)\right)+\cdots \\
& +\left(\int _ { T _ { l } ^ { * } + ( q _ { t } - 1 ) \omega } ^ { T _ { l } ^ { * } + q _ { t } \omega } \left[-\eta b_{q}(s)+\lambda b_{l}(s)+\sum_{j=l+1}^{n} \eta a_{q j}(s) x_{j}^{\theta_{q j}}(s)\right.\right. \\
& \left.\left.-\sum_{j=l+1}^{n} \lambda a_{l j}(s) x_{j}^{\theta_{l j}}(s)\right] d s+\sum_{T_{l}^{*}+\left(q_{t}-1\right) \omega \leq t_{k}<T_{l}^{*}+q_{t} \omega} \ln \left(h_{q k}^{-\eta} h_{l k}^{\lambda}\right)\right) \\
& +\left(\int _ { T _ { l } ^ { * } + q _ { t } \omega } ^ { t } \left[-\eta b_{q}(s)+\lambda b_{l}(s)+\sum_{j=l+1}^{n} \eta a_{q j}(s) x_{j}^{\theta_{q j}}(s)\right.\right. \\
& \left.\left.\left.-\sum_{j=l+1}^{n} \lambda a_{l j}(s) x_{j}^{\theta_{l j}}(s)\right] d s+\sum_{T_{l}^{*}+q_{t} \omega \leq t_{k}<t} \ln \left(h_{q k}^{-\eta} h_{l k}^{\lambda}\right)\right)\right\} \\
& \leq M_{l} \exp \left(-\frac{1}{2} \delta \eta \eta_{0} q_{t}\right),
\end{aligned}
$$

where

$$
M_{l}=V_{l}\left(T_{l}^{*}\right) \exp \left\{\left(\operatorname{wup}_{t \geq 0}\left\{\eta\left|b_{q}(t)\right|+\lambda\left|b_{l}(t)\right|+\sum_{j=l+1}^{n}\left(\eta a_{q j}(t)+\lambda a_{l j}(t)\right) \delta^{\theta_{l j}}\right\}+(\lambda+\eta) P\right)\right\},
$$

Since $q_{t} \rightarrow \infty$ as $t \rightarrow \infty$, we obtain from (3.29)

$$
V_{l}(t) \longrightarrow 0 \text { as } t \longrightarrow \infty,
$$


Since

$$
\begin{gathered}
\left(x_{l}(t)\right)^{\eta}=V_{l}(t)\left(x_{q}(t)\right)^{\lambda}, \\
\left(h_{l k} x_{l}\left(t_{k}\right)\right)^{\eta}=h_{l k}^{\eta} h_{q k}^{-\lambda} V_{l}\left(t_{k}\right)\left(h_{q k} x_{q}\left(t_{k}\right)\right)^{\lambda},
\end{gathered}
$$

by the boundedness of $x(t)$ on $[0, \infty)$, it follows that

$$
x_{l}(t) \longrightarrow 0 \text { as } t \longrightarrow \infty
$$

Finally, by the induction principle, we obtain that $x_{i}(t) \rightarrow 0$ as $t \rightarrow \infty$ for all $i>r$. This completes the proof of Theorem 3.1.

\section{Permanence}

In this section, we study the permanence of partial species $x_{i}(t)(i=1,2, \ldots, r)$ of system (1.2). We state and prove the following result.

Theorem 4.1. Suppose that all the conditions of Theorem 3.1 hold. If for each $i=1,2, \ldots, r$

$$
\liminf _{t \rightarrow \infty}\left(\int_{t}^{t+\omega}\left[b_{i}(s)-\sum_{j \neq i}^{r} a_{i j}(s) u_{j 0}^{\theta_{i j}}(t)\right] d s+\sum_{t \leq t_{k}<t+\omega} \ln h_{i k}\right)>0
$$

where $u_{i 0}$ is some fixed positive solution of (2.3), then species $x_{i}(i=1,2, \ldots, r)$ are permanent, that is, there are positive constants $m$ and $M$ such that for any positive solution $x(t)=$ $\left(x_{1}(t), x_{2}(t), \ldots, x_{n}(t)\right)$ of system $(1.2)$

$$
m \leq \liminf _{t \rightarrow \infty} x_{i}(t) \leq \limsup _{t \rightarrow \infty} x_{i}(t) \leq M, \quad i=1,2, \ldots, r
$$


Proof. From (4.1) and the boundedness of functions $a_{i j}(t)(i, j=1,2, \ldots, n)$ on $R_{+}$, there are constants $\varepsilon_{0}>0$ and $T_{1}>0$ such that for any $t \geq T_{1}$ and $i=1,2, \ldots, r$.

$$
\int_{t}^{t+\omega}\left[b_{i}(s)-\sum_{j=1}^{n} a_{i j}(s) \varepsilon_{0}-\sum_{j \neq i}^{r} a_{i j}(s) u_{j 0}^{\theta_{i j}}(s)\right] d s+\sum_{t \leq t_{k}<t+\omega} \ln h_{i k}>\varepsilon_{0} .
$$

For any $i \leq r$, from system (1.2), we have

$$
\begin{aligned}
\frac{d x_{i}(t)}{d t} & \leq x_{i}(t)\left[b_{i}(t)-\sum_{j=1}^{n} a_{i j}(t) x_{j}^{\theta_{i j}}(t)\right], \\
& \leq x_{i}(t)\left[a_{i}(t)-b_{i i}(t) x_{i}^{\theta_{i i}}(t)\right], \quad t \neq t_{k}, \quad t \geq 0, \\
x_{i}\left(t_{k}^{+}\right) & =h_{i k} x_{i}\left(t_{k}\right), \quad k=1,2, \ldots,
\end{aligned}
$$

we have

$$
x_{i}(t) \leq u_{i}(t) \quad \forall t \geq 0,
$$

where $u_{i}(t)$ is the solution of (2.3) with initial condition $u_{i}(0) \geq x_{i}(0)$. From Lemma 2.1 and Theorem 3.1, for the above constant $\varepsilon_{0}$ there is a $T_{2} \geq T_{1}$ such that for all $t \geq T_{2}$

$$
\begin{gathered}
x_{i}(t) \leq u_{i}(t) \leq u_{i 0}(t)+\varepsilon_{0}, \quad i=1,2, \ldots, r, \\
x_{i}(t)<\varepsilon_{0}, \quad i=r+1, r+2, \cdots, n .
\end{gathered}
$$

Let

$$
\begin{gathered}
\gamma_{i}=\sup _{t \geq 0}\left\{\left|b_{i}(t)\right|+\sum_{j=1}^{n} a_{i j}(t) \varepsilon_{0}+\sum_{j \neq i}^{r} a_{i j}(t) u_{j 0}^{\theta_{i j}}(t)\right\}, \\
m=\min _{1 \leq i \leq r}\left\{\varepsilon_{0} \exp \left(-\gamma_{i} \omega-P\right)\right\},
\end{gathered}
$$

where constant $P>0$ is given in (2.7). Obviously, $m>0$ and $m$ is independent of any positive solution of system (1.2).

Now, we prove that there is a $T_{3} \geq T_{2}$ such that

$$
x_{i}(t) \geq m \quad \forall t \geq T_{3}, i=1,2, \ldots, r .
$$


We only need to consider the following three cases for each $i=1,2, \ldots, r$.

Case I. There is a $t_{1} \geq T_{2}$ such that $x_{i}(t) \leq \varepsilon_{0}^{\prime}=\sqrt[\theta_{i i}]{\varepsilon_{0}}$ for all $t \geq t_{1}$.

Case II. There is a $t_{2} \geq T_{2}$ such that $x_{i}(t) \geq \varepsilon_{0}^{\prime}$ for all $t \geq t_{2}$.

Case III. $x_{i}(t)$ oscillates about $\varepsilon_{0}^{\prime}$ for all $t \geq T_{2}$.

For Case I, let $t=t_{1}+l \omega$, where $l \geq 0$ is any integer. From (4.3)-(4.7) we obtain

$$
\begin{gathered}
x_{i}(t)=x_{i}\left(t_{1}\right) \exp \left(\int_{t_{1}}^{t}\left(b_{i}(s)-a_{i i}(s) x_{i}^{\theta_{i i}}(s)-\sum_{j \neq i}^{n} a_{i j}(s) x_{j}^{\theta_{i j}}(s)\right) d s+\sum_{t_{1} \leq t_{k}<t} \ln h_{i k}\right) \\
\geq x_{i}\left(t_{1}\right) \exp \left(\int_{t_{1}}^{t_{1}+\omega}\left(b_{i}(s)-\sum_{j=1}^{n} a_{i j}(s) \varepsilon_{0}-\sum_{j \neq i}^{r} a_{i j}(s) u_{j 0}^{\theta_{i j}}(s)\right) d s\right. \\
+\sum_{t_{1} \leq t_{k}<t_{1}+\omega} \ln h_{i k}+\cdots+\int_{t_{1}+(l-1) \omega}^{t_{1}+l \omega}\left(b_{i}(s)-\sum_{j=1}^{n} a_{i j}(s) \varepsilon_{0}-\sum_{j \neq i}^{r} a_{i j}(s) u_{j 0}^{\theta_{i j}}(s)\right) d s \\
\left.+\sum_{t_{1}+(l-1) \omega \leq t_{k}<t_{1}+l \omega} \ln h_{i k}\right) \\
\geq x_{i}\left(t_{1}\right) \exp \left(l \varepsilon_{0}\right) .
\end{gathered}
$$

Therefore, $x_{i}(t) \rightarrow \infty$ as $l \rightarrow \infty$ which leads to a contradiction.

For Case III, we choose two sequences $\left\{\rho_{n}\right\}$ and $\left\{\rho_{n}^{*}\right\}$ satisfying $T_{2} \leq \rho_{1}<\rho_{1}^{*}<\cdots<$ $\rho_{n}<\rho_{n}^{*}<\cdots$ and $\lim _{n \rightarrow \infty} \rho_{n}=\lim _{n \rightarrow \infty} \rho_{n}^{*}=\infty$ such that

$$
\begin{gathered}
x_{i}\left(\rho_{n}\right) \geq \varepsilon_{0}^{\prime}, \quad x_{i}\left(\rho_{n}^{+}\right) \leq \varepsilon_{0}^{\prime}, \quad x_{i}\left(\rho_{n}^{*}\right) \leq \varepsilon_{0}^{\prime}, \quad x_{i}\left(\rho_{n}^{*^{+}}\right) \geq \varepsilon_{0}^{\prime}, \\
x_{i}(t) \leq \varepsilon_{0}^{\prime} \quad \forall t \in\left(\rho_{n}, \rho_{n}^{*}\right), \\
x_{i}(t) \geq \varepsilon_{0}^{\prime} \quad \forall t \in\left(\rho_{n}^{*}, \rho_{n+1}\right) .
\end{gathered}
$$

For any $t \geq T_{2}$, if $t \in\left(\rho_{n}, \rho_{n}^{*}\right]$ for some integer $n$, then we can choose an integer $l \geq 0$ such that $t=\rho_{n}+l \omega+v_{i}$, where $v_{i} \in[0, \omega)$ is a constant. Since for any $t \in\left(\rho_{n}, \rho_{n}^{*}\right)$ from (4.6) and (4.7) we have

$$
\dot{x}_{i}(t) \geq x_{i}(t)\left(b_{i}(t)-\sum_{j=1}^{n} a_{i j}(t) \varepsilon_{0}-\sum_{j \neq i}^{r} b_{i j}(t) u_{j 0}^{\theta_{i j}}(t)\right), \quad t \neq t_{k} .
$$


Integrating this inequality from $\rho_{n}$ to $t$, then from (4.7) and (3.28)-(3.29) we have

$$
\begin{gathered}
x_{i}(t) \geq x\left(\rho_{n}\right) \exp \left(\int_{\rho_{n}}^{t}\left(b_{i}(s)-\sum_{j=1}^{n} a_{i j}(s) \varepsilon_{0}-\sum_{j \neq i}^{r} a_{i j}(s) u_{j 0}^{\theta_{i j}}(s)\right) d s+\sum_{\rho_{n} \leq t_{k}<t} \ln h_{i k}\right) \\
\geq \varepsilon_{0} \exp \left(\int_{\rho_{n}}^{\rho_{n}+\omega}\left(b_{i}(s)-\sum_{j=1}^{n} a_{i j}(s) \varepsilon_{0}-\sum_{j \neq i}^{r} a_{i j}(s) u_{j 0}^{\theta_{i j}}(s)\right) d s\right. \\
+\sum_{\rho_{n} \leq t_{k}<\rho_{n}+\omega} \ln h_{i k}+\cdots+\int_{\rho_{n}+(l-1) \omega}^{\rho_{n}+l \omega}\left(b_{i}(s)-\sum_{j=1}^{n} a_{i j}(s) \varepsilon_{0}-\sum_{j \neq i}^{r} a_{i j}(s) u_{j 0}^{\theta_{i j}}(s)\right) d s \\
\left.+\sum_{\rho_{n}+(l-1) \omega \leq t_{k}<\rho_{n}+l \omega} \ln h_{i k}\right) \\
+\int_{\rho_{n}+l \omega}^{\rho_{n}+l \omega+v_{i}}\left(b_{i}(s)-\sum_{j=1}^{n} a_{i j}(s) \varepsilon_{0}-\sum_{j \neq i}^{r} a_{i j}(s) u_{j 0}^{\theta_{i j}}(s)\right) d s+\sum_{\rho_{n}+l \omega \leq t_{k}<\rho_{n}+l \omega+v_{i}} \ln h_{i k} \\
\geq \varepsilon_{0} \exp \left(-\gamma_{i} \omega-P\right) .
\end{gathered}
$$

If there exists an integer $n$ such that $t \in\left(\rho_{n}^{*}, \rho_{n+1}\right]$, then we obviously have

$$
x_{i}(t) \geq \varepsilon_{0}>\varepsilon_{0} \exp \left(-\gamma_{i} \omega-P\right) .
$$

This shows that for Case III we always have

$$
x_{i}(t) \geq \varepsilon_{0} \exp \left(-\gamma_{i} \omega-P\right), \quad \forall t \geq T_{2} .
$$

Finally, if Case II holds, then from $x_{i}(t) \geq \varepsilon_{0}^{\prime}$ for all $t \geq t_{1}$, we can directly obtain that (4.9) holds.

Therefore, from Lemma 2.2 and (4.9), it follows that species $x_{i}(t)(i=1,2, \ldots, r)$ are permanent. This proof of Theorem 4.1 is completed.

\section{Global Attractivity}

In this section, we further discuss the global attractivity of species $x_{i}(t)(i \leq r)$. In order to obtain our results, we first consider the following subsystem which is composed of the species 
$x_{i}(t)(i \leq r)$ of system (1.2) and for convenience of statement we use the variable $u_{i}(t)(i \leq r)$ to denote the species of this subsystem,

$$
\begin{aligned}
& \frac{d u_{i}(t)}{d t}=u_{i}(t)\left[b_{i}(t)-\sum_{j=1}^{r} a_{i j}(t) u_{j}^{\theta_{i j}}(t)\right], \quad t \neq t_{k}, \\
& u_{i}\left(t_{k}^{+}\right)=h_{i k} u_{i}\left(t_{k}\right), \quad i=1,2, \ldots, r, k=1,2, \ldots
\end{aligned}
$$

We need the following lemma.

Lemma 5.1. Suppose that assumption $H$ and condition (4.1) of Theorem 4.1 hold. Then subsystem (5.1) is permanent.

Lemma 5.1 can be proved by using the same method given in the proof of Theorem 4.1. We now state and prove the main result of this section.

Theorem 5.2. Suppose that all conditions of Theorem 3.1 and Theorem 4.1 hold. If there are positive constants $\rho, D$ and $d_{i}(i=1,2, \ldots, r)$ and nonnegative integrable function $\mu(t)$ defined on $R_{+}$, satisfying $\int_{s}^{t} \mu(\tau) d \tau \geq-D+\rho(t-s)$ for all $t \geq s \geq 0$, such that

$$
d_{i} a_{i i}(t)-\sum_{j \neq i}^{r} d_{j} a_{j i}(t) \geq \mu(t), \quad i=1,2, \ldots, r
$$

for all $t \geq 0$, then for any positive solution $x(t)=\left(x_{1}(t), x_{2}(t), \ldots, x_{n}(t)\right)$ of system (1.2) and any positive solution $u(t)=\left(u_{1}(t), u_{2}(t), \ldots, u_{r}(t)\right)$ of subsystem (5.1)

$$
\lim _{t \rightarrow \infty}\left(x_{i}(t)-u_{i}(t)\right)=0, \quad i=1,2, \ldots, r
$$

Proof. Let $x(t)=\left(x_{1}(t), x_{2}(t), \ldots, x_{n}(t)\right)$ be a positive solution of system (1.2) and $u(t)=$ $\left(u_{1}(t), u_{2}(t), \ldots, u_{r}(t)\right)$ be a positive solution of subsystem (5.1). By Theorem 3.1, we have $x_{i}(t) \rightarrow 0$ as $t \rightarrow \infty$ for all $i>r$. From Theorem 4.1 and Lemma 5.1, there are positive constants $m$ and $M$ such that

$$
m \leq x_{i}(t), \quad u_{i}(t) \leq M, \quad i=1,2, \ldots, r,
$$

for all $t \geq 0$. Choose the Liapunov function as follows:

$$
V_{r}(t)=\sum_{i=1}^{r} d_{i}\left|\ln x_{i}(t)-\ln u_{i}(t)\right|
$$


Since

$$
\begin{aligned}
V_{r}\left(t_{k}^{+}\right) & =\sum_{i=1}^{r} d_{i}\left|\ln x_{i}\left(t_{k}^{+}\right)-\ln u_{i}\left(t_{k}^{+}\right)\right| \\
& =\sum_{i=1}^{r} d_{i}\left|\ln h_{i k} x_{i}\left(t_{k}\right)-\ln h_{i k} u_{i}\left(t_{k}\right)\right| \\
& =V_{r}\left(t_{k}\right),
\end{aligned}
$$

then $V(t)$ is continuous for all $t \geq 0$. Calculating the upper right derivative of $V_{r}(t)$, we have

$$
\begin{aligned}
D^{+} V_{r}(t) & \leq \sum_{i=1}^{r} d_{i}\left(-a_{i i}\left|x_{i}^{\theta_{i i}}(t)-u_{i}^{\theta_{i i}}(t)\right|+\sum_{j \neq i}^{r} a_{i j}(t)\left|x_{j}^{\theta_{i j}}(t)-u_{j}^{\theta_{i j}}(t)\right|\right)+g(t) \\
& =-\sum_{i=1}^{r}\left(d_{i} a_{i i}-\sum_{j \neq i}^{r} d_{j} a_{j i}(t)\right)\left|x_{i}^{\theta_{j i}}(t)-u_{i}^{\theta_{j i}}(t)\right|+g(t),
\end{aligned}
$$

for all $t \geq 0$, where

$$
g(t)=\sum_{i=1}^{r} d_{i} \sum_{j=r+1}^{n} a_{j i}(t) x_{j}^{\theta_{j i}}(t) .
$$

By (5.2), we have

$$
D^{+} V_{r}(t) \leq-\mu(t) \sum_{i=1}^{r}\left|x_{i}^{\theta_{j i}}(t)-u_{i}^{\theta_{j i}}(t)\right|+g(t), \quad \forall t \geq 0
$$

By (5.4), we further obtain

$$
D^{+} V_{r}(t) \leq-\lambda \mu(t) V_{r}(t)+g(t), \quad \forall t \geq 0,
$$

where $\lambda=\min _{1 \leq i \leq r} d_{i}^{-1} m>0$. Applying the comparison theorem and the variation of constants formula of first-order linear differential equation, we have

$$
V_{r}(t) \leq e^{-\int_{0}^{t} \lambda \mu(s) d s}\left(\int_{0}^{t} g(s) e^{\int_{0}^{s} \lambda \mu(\tau) d \tau} d s+V_{r}(0)\right)
$$

for all $t \geq 0$. Since $g(t) \rightarrow 0$ as $t \rightarrow \infty$, from the properties of function $\mu(t)$ and (5.11), it is not hard to obtain $V_{r}(t) \rightarrow 0$ as $t \rightarrow \infty$. That shows

$$
\lim _{t \rightarrow \infty}\left(x_{i}(t)-u_{i}(t)\right)=0, \quad i=1,2, \ldots, r .
$$

This completes the proof of Theorem 5.2. 


\section{References}

[1] J. Hou, Z. Teng, and S. Gao, "Permanence and global stability for nonautonomous N-species LotkaValterra competitive system with impulses," Nonlinear Analysis, vol. 11, no. 3, pp. 1882-1896, 2010.

[2] J. Hou, Z. D. Teng, and S. J. Gao, "Partial extinction, permanence and global attractivity in nonautonomous $n$-species Lotka-Volterra competitive systems with impulses," Applied Mathematics: A Journal of Chinese Universities, vol. 26, no. 2, pp. 161-176, 2011.

[3] M. He, Z. Li, and F. Chen, "Permanence, extinction and global attractivity of the periodic Gilpin-Ayala competition system with impulses," Nonlinear Analysis, vol. 11, no. 3, pp. 1537-1551, 2010. 


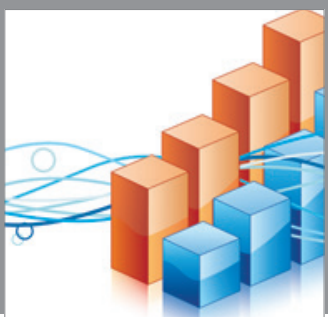

Advances in

Operations Research

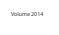

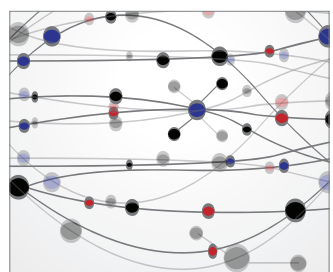

\section{The Scientific} World Journal
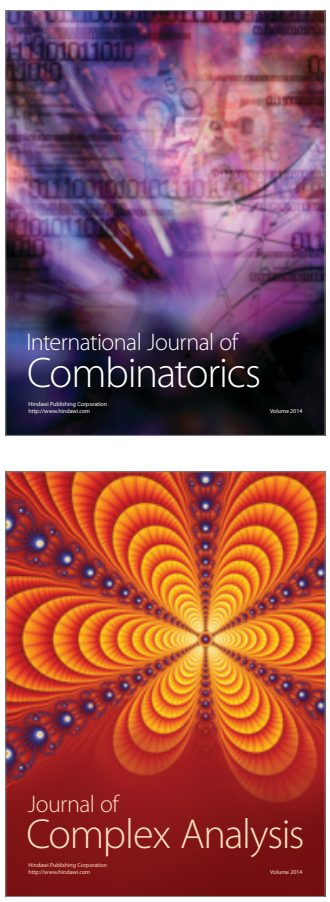

International Journal of

Mathematics and

Mathematical

Sciences
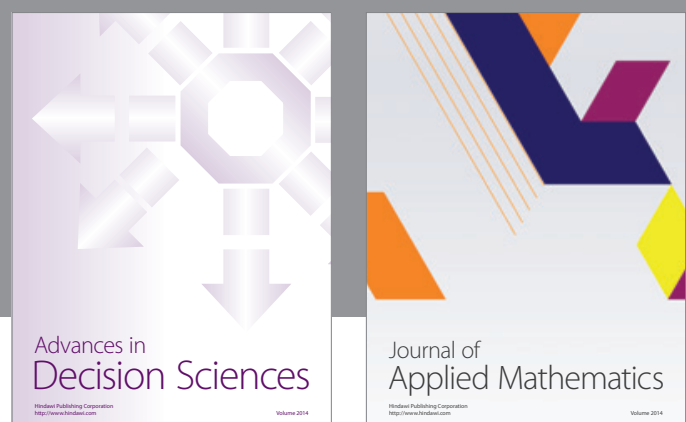

Journal of

Applied Mathematics
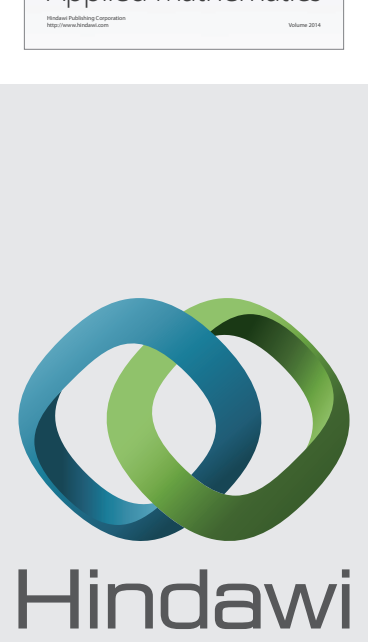

Submit your manuscripts at http://www.hindawi.com
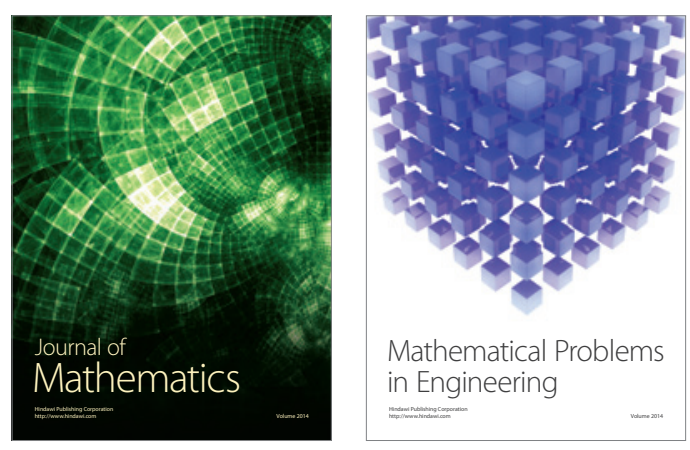

Mathematical Problems in Engineering
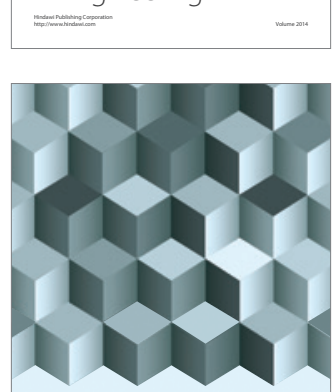

Journal of

Function Spaces
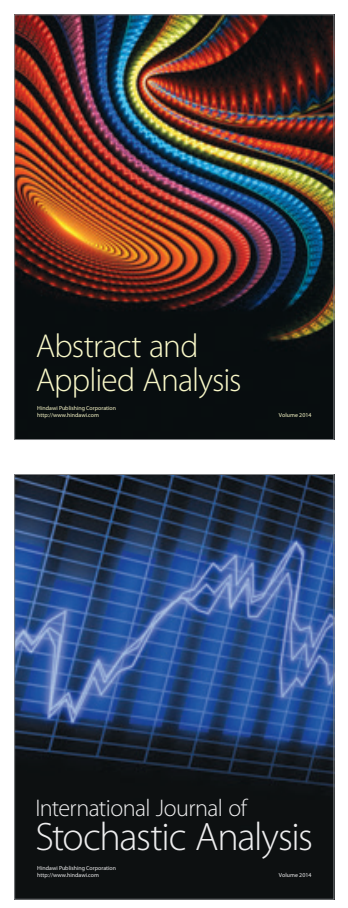

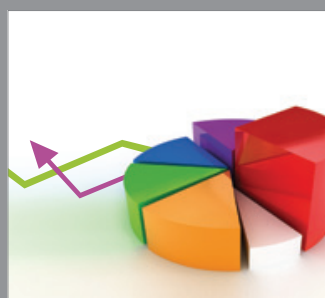

ournal of

Probability and Statistics

Promensencen
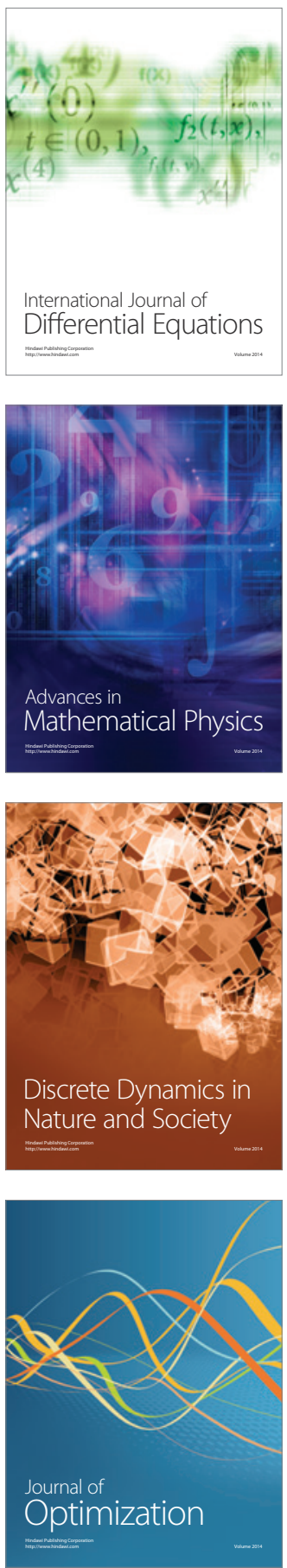